\title{
Artificial Intelligence: Demystifying Dentistry - The Future and Beyond
}

\author{
Tejaswi Katne ${ }^{1}$, Alekhya Kanaparthi ${ }^{2}$, Srikanth Gotoor ${ }^{3}$, Srikar Muppirala ${ }^{4}$, Ramaraju Devaraju ${ }^{5}$, Ramlal Gantala ${ }^{6}$ \\ ${ }^{1}$ Consultant Oral and Maxillofacial Radiologist Max3G Imaging, Hyderabad, Telangana, ${ }^{2}$ Assistant Professor, Department \\ of Oral Medicine and Radiology, MNR Dental College and Hospital, Sanga Reddy, Telangana, ${ }^{3}$ Reader, Department of Oral \\ Medicine and Radiology, SVS Institute of Dental Sciences, Mahabubangar, Telangana, ${ }^{4}$ Consultant Oral and Maxillofacial \\ Radiologist, Vijaya Diagnostic Centre, Hyderabad, Telangana, ${ }^{5}$ Honorary Professor, Department of Oral Medicine and \\ Radiology, SVS Institute of Dental Sciences, Mahabubnagar, Telangana, ${ }^{6}$ Professor and HOD, Department of Oral Medicine \\ and Radiology, SVS Institute of dental sciences, Mahabubnagar, Telangana, India
}

Corresponding author: Tejaswi Katne, Flat No 102, Sri Shailaja Castle Appartments, Beside More Super Market and Cooperative Bank, Saidabad Colony Main Road, Saidabad, Hyderabad 500059, India

DOI: http://dx.doi.org/10.21276/ijcmsr.2019.4.4.2

How to cite this article: Tejaswi Katne, Alekhya Kanaparthi, Srikanth Gotoor, Srikar Muppirala, Ramaraju Devaraju, Ramlal Gantala. Artificial intelligence: demystifying dentistry - the future and beyond. International Journal of Contemporary Medicine Surgery and Radiology. 2019;4(4):D6-D9.

\section{A B S T R A C T}

A plethora of advancements in the field of technology during the last few decades have integrated these technological advancements in our day to day life. Artificial intelligence $(\mathrm{Al})$ is a field of engineering science dealing with the computational understanding and ability of the computers to mimic human brain to exhibit an intelligent behaviour to perform the tasks effortlessly. It has begun to establish itself even into the field of dentistry and medicine. Right from data acquisition to even performing virtual surgeries were made possible with the introduction of virtual reality in the medicine and dentistry. The need for proper documentation of the patient's information, quick and reliable treatment protocols throughrobotics in the field of surgery has encouraged the use of these software technologies in assisting the dentist to diagnose and treat the patients efficiently. However, this technological advancement is still in the stages of infancy and this article is an attempt to highlight the role of artificial intelligence in dentistry.

Keywords: Artificial Intelligence, Deep Learning, Virtual Reality, Dentistry, Robotics and Virtual Surgeries

\section{INTRODUCTION}

Technological advancements have revolutionized the field of medicine and dentistry within the last few decades. Newer technologies have been consistently developed based on the principles that try to mimic the human brain functioning to develop solutions that do not just follow pre programmed instructions, but also have some traits of the human's such as the reasoning ability and experienced learning from practice without being programmed manually. Artificial intelligence (AI) is defined as the study of intelligent agents, any device that perceives its environment and takes action that maximize its chance of successfully achieving its goals (Russell and Norvig 2003). ${ }^{1}$

The applications of these Artificial Intelligence technologies in non medical fields like telecommunication may include Gaming, language processing, Robotics and Image recognition. Artificial intelligence in the field of medicine and dentistry benefitted healthcare professionals, to improve patient healthcare services and may serve as a great tool.

\section{How does this work?}

A machine learning prediction technique is an algorithm that estimates an unknown dependency between a set of given input variables and its output variable. When such dependency is observed, it can predict the future output by identifying the target function that best describes the behaviour governing the input-output patterns. ${ }^{2}$

\section{Clinical decision support system CDSS}

A Clinical decision support system (CDSS) is a network between an extensive dynamic (medical) knowledge database and an inferencing output mechanism that are a set of algorithms derived from evidence-based medical practice implemented through medical logic modules. Currently, the interactive interphase with voice controls are designed to assist the health care professional to work more efficiently with time saving and cost effective clinical dental practice. ${ }^{3}$

\section{Artificial Neural network (ANN)}

Neural networks are mathematical computing models that simulate the functioning of the human brain. It is a system made of several interconnected processing elements that process data by their automated dynamic-state response to external inputs. Neural networks have wide range of features that make them suitable for a variety of tasks in medical and dental diagnostics. ${ }^{4}$

At present; neural networks are used as principal solutions for various problems like classification, approximation, pattern recognition, image matching, risk prediction and memory 
simulation. Neural network expert systems may be trained with clinical data and these can be used in clinical situations of diagnostic dilemmas to improve clinical strategies of health care systems. ${ }^{4}$

\section{Augmented reality and virtual reality}

Dentistry is a skill based profession with tremendous amount of knowledge. Augmented reality uses computergenerated images that are superimposed a on a user's view. ${ }^{5}$ The invention of augmented reality has aids in meeting the patient's expectations and also helps the dentist to exactly understand the needs of the patient. Cosmetic dentistry is one such branch of dentistry where, the treatment outcome can be virtually demonstrated to the patient. This not only motivates the patient but also guides the dentist to focus on the predetermined outcome.

Virtual reality is a computer-generated simulation of a three dimensional models that can be interacted with, in a seemingly real environment. The AI systems along with virtual reality has been used not only as an education tool but also as a methods of distraction for non-pharmacological control of pain. ${ }^{5}$

\section{APPLICATIONS OF ARTIFICIAL INTELLIGENCE IN VARIOUS FIELDS OF DENTISTRY}

General Dentistry: The elegance of AI is that machines can be trained to evaluate large data sets and memorize from them to impart most favourable diagnoses. Artificial intelligence based virtual dental companions can perform different tasks in routine dental practice with less man power, fewer errors and with greater precision compared to humans. It guides in coordinating appointments, executing insurance and paperworks besides assisting clinical diagnosis or treatment planning. ${ }^{5}$ It also helps in alarming the dental practioner about subjects medical history as well as habits like smoking and alcoholism. Especially in dental emergencies when the practioner is unavailable the patient has a option of emergency teleassistance which would benefit the patient. ${ }^{6}$

AI has now become a part of our daily lives..... with advent of siri, Alexa, we are now used to voice commands. The dental practice now has also been updated from use of dental chairs that were touch sensitive to voice controlled dental chairs that do not need any manual input from clinician. ${ }^{7}$ Based on the voice command the chair positions, water dispensing and light control can be efficiently handled. Furthermore, relatively sterile form of clinical examination can be practiced with reduced risk of cross contamination during treatments. Thus a detailed virtual database of the patient can be created which will go a long way in providing ideal treatment for the patient.

Oral and Maxillofacial Surgery: The tremendous utilization of artificial intelligence in oral and maxillofacial surgery is alongside the evolution of robotic surgery where human body motion and human intelligence is simulated. ${ }^{8}$

AI software programs has assisted the surgeon in planning surgeries with reduced operation time there by preserving the vital structures around to the smallest detail prior to the actual surgery with higher intra operative accuracy. ${ }^{9}$ One more fruitful clinical application is image guided surgery that confess further accurate surgical resection possibly decreasing need for revision procedures.

Bio printing is the other ingenious applications of AI, where organs and living tissues can be formulated in successive thin layers of cells which in the future may be used for regeneration of oral hard and soft tissues that are lost due to pathological or accidental reasons. ${ }^{10}$

Oral medicine: Artificial intelligence can be used as a useful modality in diagnosis and treatment of lesions of oral cavity and can be employed in screening and classifying suspicious altered mucosa undergoing premalignant and malignant changes. ${ }^{11,12}$ Artificial intelligence might accurately predict a genetic predisposition for oral cancer for a large population. ${ }^{12}$ The use of ANNs in the diagnoses subtypes of temporomandibular disorders has been studied by Bas B et al. They suggested that ANNs may act as an adjuvant diagnostic tool for dentist. ${ }^{13}$

Dental and Maxillofacial Radiology: Radiologists are primarily known for their image interpretation skills. Advanced breakthroughs in image recognition using artificial intelligence Systems has shifted from science fable into reality in the radiology practice in the last two decades. In head and neck imaging modalities AI provides added advantage owing to its distinctive ability to learn and can be assimilated with other imaging modalities like CBCT, MRI to determine infinitesimal deviations from normality that could have gone unrecognized with human eye. Illustrations include definite location of landmarks on radiographs aids in location of minor apical foramen ${ }^{14}$, detection of vertical root fractures ${ }^{15}$, Cephalometric analysis, thereby strengthening the accuracy of working length determination, Logicon Caries Detector helps in detection and characterization of proximal caries. ${ }^{16,17}$ Economically all these could be translated in to a better patient care.

Forensic Odontology: Age estimation is an important aspect of forensic odontology that has been used over decades for various reasons. Human age estimation using the facial images have been under tremendous research in the recent past. $^{18}$

However, the information regarding the use of artificial intelligence in dental Age determination is sparse. Dental age estimation is based on the sequence of tooth formation and eruption time. Stages of Tooth formation can be observed radio graphically. Radiographic methods of Age estimation is commonly used due to the availability of the pre and post treatment records and also the ease of the availability of the data that can be retrieved any time. With the advent of the artificial intelligence, there are several programming neural networks can train the computers to automatically to estimate the age.

Dental Education System: Clinical dentistry is an essential part of dentistry for learning the skills, to enhance patient care. Traditionally, pre-clinical operative training for dental students was combination of theoretical teaching and practical learning. Recently artificial intelligence has been incorporated into tutoring intelligent education system and 
training in dentistry. ${ }^{6}$

These technologies have the ability to create virtual reality that enables simulation of the practical procedures in three dimensions that enable simulation and allow assess to clinical and surgical techniques. ${ }^{19}$ The practice sessions can be done several times till the skill set is expertise by students over the subject prior to actual handling of real clinical cases reducing the risk of iatrogenic damage. This method of training proves to be more efficient, inexpensive and reliable.

Prosthodontics: AI combined with designing softwares can aid the dentist to design the best possible and aesthetic prostheses considering number of factors like facial measurements, anthropological calculations, ethnicity and patient desire.AI plays a major role in identifying the type of bone, cortical thickness for making precise surgical guides for pacing implants. ${ }^{20}$ Another breakthrough in this field is the use of CADCAM technology ${ }^{21}$ which creates a $2 \mathrm{D}$ and $3 \mathrm{D}$ models has replaced the time consuming and laborious process of conventional casting thereby reducing human errors.

Virtual reality simulation (VRS) technology can be used to simulate the facial profiles post treatment. This not only enables the dentist to efficiently design the esthetics, but also acts as a motivational tool for the patient. ${ }^{22}$

Orthodontics: Future of orthodontics is no longer driven by appliances but by AI. In the recent times by analysing the radiographs and photographs AI helps in orthodontic diagnosis, planning treatment and monitoring the progression of treatment. ${ }^{24}$ With the advent of intraoral scanners and cameras making of dental impression is being replaced by digital impressions ${ }^{25}$ and the above data is fed in to the system the set algorithms and AI software helps in predicting tooth movements and final outcome of the treatment ${ }^{26}$ Latest technologies combined with customised aligner based orthodontics can improve case acceptance.

Periodontics: Deep learning analysis using radiographs can assist in diagnosing and treatment planning of periodontal diseases by enabling the early detection of periodontal changes, ${ }^{27}$ boneloss, and changes in bone density. Detection of peri implantitis can also help in early intervention in implantology. ${ }^{28}$

\section{CONCLUSION}

The field of artificial intelligence has transformed medicine and dentistry in several ways. Though Artificial Intelligence systems are a great asset in dentistry and dental education, the human biological system is complex and it is to be noted that these technological advancements are still the brain child of innovations and discoveries by mankind. Furthermore, AI can only assist the clinician in performing the tasks efficiently, but in no way replace the intellect of the human knowledge, skill and treatment planning.

\section{REFERENCES}

1. Russel S, Norvig P. Artificial Intelligence: A Modern Approach. $3^{\text {rd }}$ ed. New Jersey: Pearson Education; 2010

2. Kalappanavar A, Sneha S, Annigeri RG. Artificial intelligence: A dentist's perspective. Journal of Medicine,
Radiology, Pathology and Surgery. 2018;5(2):2-4.

3. Wulff A, Montag S, Steiner B, Marschollek M, Beerbaum P, Karch A et al.CADDIE2-evaluation of a clinical decision-support system for early detection of systemic inflammatory response syndrome in paediatric intensive care: study protocol for a diagnostic study. BMJ open. 2019;9(6):e028953.

4. Brickley MR, Shepherd JP, Armstrong RA. Neural networks: A new technique for development of decision support systems in dentistry.J Dent 1998;26(4):305-9.

5. Khanna SS, Dhaimade PA. Artificial Intelligence: Transforming dentistry today. Indian J Basic Appl Med Res 2017;6(4):161-7.

6. Feeney L, Reynolds PA, Eaton KA, Harper J. A description of the new technologies used in transforming dental education. British Dental Journal 2008;204(2): 19-28.

7. Deshmukh SV. Artificial intelligence in dentistry. J Int Clin Dent Res Organ 2018;10(3):47-8

8. Ruppin J, Popovic A, Strauss M, Spüntrup E, Steiner A, Stoll C. Evaluation of the accuracy of three different computer-aided surgery systems in dental implantology: optical tracking vs. stereolithographic splint systems. Clinical oral implants research. 2008;19(7):709-16.

9. Widmann G. Image-guided surgery and medical robotics in the cranial area. Biomedical imaging and intervention journal. 2007;3(1).

10. Seol YJ, Kang HW, Lee SJ, Atala A, Yoo JJ. Bioprinting technology and its applications. European Journal of Cardio-Thoracic Surgery. 2014;46(3):342-8.

11. Lim K, Moles DR, Downer MC, Speight PM. Opportunistic screening for oral cancer and precancer in general dental practice: results of a demonstration study. British dental journal. 2003;194(9):497.

12. Rosmai MD, Sameemii AK, Basir A, Mazlipahiv IS, Norzaidi MD. The use of artificial intelligence to identify people at risk of oral cancer: empirical evidence in Malaysian University. International Journal of Scientific Research in Education. 2010;3(1):10-20.

13. Bas B, Ozgonenel O, Ozden B, Bekcioglu B, Bulut $\mathrm{E}$, Kurt $\mathrm{M}$. Use of artificial neural network in differentiation of subgroups of temporomandibular internal derangements: a preliminary study. Journal of Oral and Maxillofacial Surgery. 2012;70(1):51-9.

14. Saghiri MA, Asgar K, Boukani KK, Lotfi M, Aghili $\mathrm{H}$, Delvarani A, et al. A new approach for locating the minor apical foramen using an artificial neural network. Int Endontic J 2012;45(4):257-65.

15. Kositbowornchai S, Plermkamon S, Tangkosol T. Performance of an artificial neural network for vertical root fracture detection: An ex vivo study. Dent Traumatol 2013;29(3):151-5.

16. Devito KL, de Souza Barbosa F, Filho WN. An artificial multilayer perceptron neural network for diagnosis of proximal dental caries. Oral Surg Oral Med Oral Pathol Oral Radiol Endod 2008;106(3):879-84.

17. Bhan A, Goyal A, Chauhan N, Wang CW. Feature line profile based automatic detection of dental caries in bitewing radiography. In 2016 International Conference on Micro-Electronics and Telecommunication Engineering (ICMETE) 2016 Sep 22 (pp. 635-640). IEEE. 
18. Kohail SN.Using artificial neural network for human age estimation based on facial images. In 2012 International Conference on Innovations in Information Technology (IIT) 2012 Mar 18 (pp. 215-219). IEEE.

19. Pohlenz P, Gröbe A, Petersik A, von Sternberg N, Pflesser B, Pommert A, et al. Virtual dental surgery as a new educational tool in dental school. Journal of CranioMaxillofacial Surgery 2010; 38(3): 560-564.

20. Vera V, Corchado E, Redondo R, Sedano J, Garcia AE. Applying soft computing techniques to optimise a dental milling process. Neurocomputing. 2013;109(2):94-104

21. Vecsei B, Joós-Kovács G, Borbély J, Hermann P. Comparison of the accuracy of direct and indirect three-dimensional digitizing processes for $\mathrm{CAD} / \mathrm{CAM}$ systems-an in vitro study. Journal of prosthodontic research. 2017;61(2):177-84.

22. Kikuchi H, Ikeda M, Araki K. Evaluation of a virtual reality simulation system for porcelain fused to metal crown preparation at Tokyo Medical and Dental University. Journal of dental education. 2013;77(6):78292

23. Sims-Williams J H, Brown I D, Matthewman A, Stephens CD; A computer controlled expert system for orthodontic advice. British Dental Journal, 1987;163(3): 161-166 29.

24. Xie X; Wang L; Wang A. Artificial Neural Network Modeling for Deciding if Extractions Are Necessary Prior to Orthodontic Treatment Angle Orthod.; 2010, 80:262-266. 30.

25. Birnbaum NS, Aaronson HB. Dental impressions using 3D digital scanners: virtual becomes reality. Compendium of Continuing Education in Dentistry 2008; 29(3): 494, 496, 498- 505.

26. Mackin N., Sims-Williams J H., Stephens C D. Artificial intelligence in dental surgery: an orthodontic expert system, a dental tool of tomorrow. 1991, Dental Update 18: 341-343.

27. Furman E, Jasinevicius TR, Bissada NF, Victoroff KZ, Skillicorn R, Buchner M. Virtual reality distraction for pain control during periodontal scaling and root planing procedures. Journal of American Dental Association 2009; 140(4): 1508-1516.

28. Sohmura T, Kusumoto N, Otani T, Yamada S, Wakabayashi K, Yatani H. CAD/CAM fabrication and clinical application of surgical template and bone model in oral implant surgery. Clinical Oral Implants Research 2009; 20(3): 87-93

Source of Support: Nil; Conflict of Interest: None

Submitted: 19-08-2019; Accepted: 20-09-2019; Published online: 14-10-2019 